\title{
Low bone mass in Sheehan's syndrome: prevalence and management
}

\author{
M. Chihaoui, D. Ben Salah, M. Yazidi, F. Chaker, B. Ftouhi, H. Slimane
}

Department of Endocrinology, University hospital la rabta, Tunis, Tunisia

\section{Introduction}

Hypopituitarism is a known cause of bone mineral loss. Our study aimed to evaluate the frequency of osteopenia and osteoporosis in patients with Sheehan's syndrome and to evaluate its management.

\section{Subjects and methods}

- Retrospective longitudinal study

- 60 cases of Sheehan's syndrome that have had a bone mineral density measurement.

- We collected:

-the parameters of osteodensitometry (dual x-ray absorptiometry, Lunar Prodigyn) at two sites; the lumbar spine L1-L4 and the femoral neck

- the received treatment,

- eventual bone fracture and

- the follow up data.

- The baseline parameters of osteodensitometry were compared to those of sixty age-, height- and weightmatched healthy control women.

- Osteopenia was defined by a T score between -2.5 and 1.0 SD and osteoporosis by a T score <-2.5 SD

\section{Results}

- The mean age at osteodensitometry measurement was $49.4 \pm 9.9$ years (range $25-76$ years).

- The mean duration of Sheehan's syndrome was 19.3 \pm 8.5 years (range 3-41 years).

- Osteopenia was present in 25 patients (41.7\%) and osteoporosis in 23 (38.3\%) (figure 1).

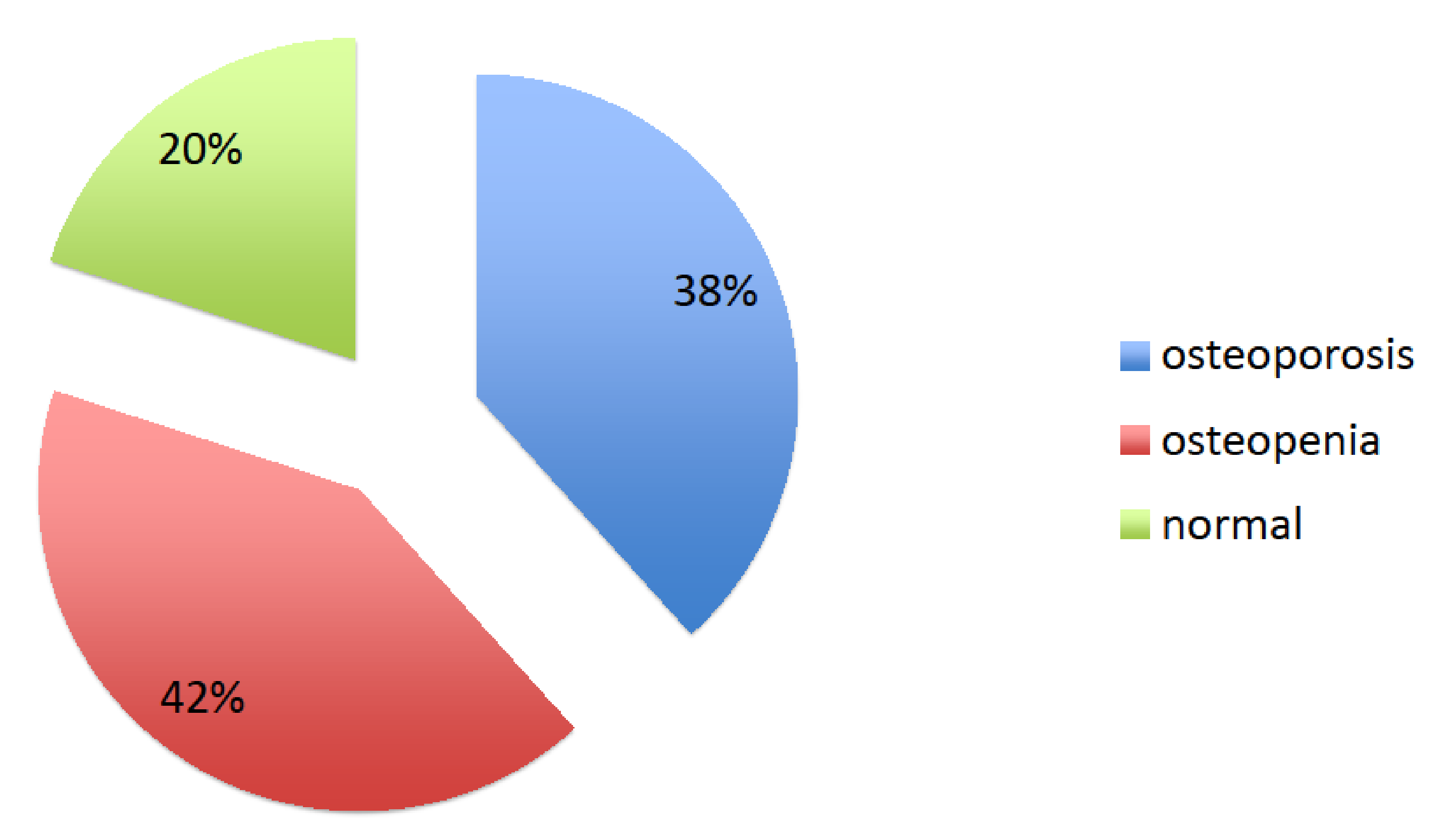

Figure 1: Frequency of osteoporosis and osteopenia

Bone mineral density was significantly lower than in the control group $(p<0.001)$ (table 1$)$.
Table 1: Comparison of age, anthropometric parameters and BMD between patients with Sheehan's syndrome and controls

\begin{tabular}{lccc}
\hline & $\begin{array}{c}\text { Patients with Shechan's } \\
\text { syndrome }(n=60)\end{array}$ & $\begin{array}{c}\text { Controls } \\
(n=60)\end{array}$ & p \\
& $49.4 \pm 9.9$ & $49.4 \pm 9.6$ & NS \\
\hline Age (years) & $153.9 \pm 5.5$ & $154.1 \pm 5.6$ & NS \\
Height (cm) & $27.2 \pm 4.9$ & $28.1 \pm 5.7$ & NS \\
BMI (Kg/m $\left.{ }^{2}\right)$ & $0,989 \pm 0,168$ & $1,121 \pm 0,156$ & $<0,001$ \\
L1-L4 BMD (g/cm $\left.{ }^{2}\right)$ & $-1,71 \pm 1,41$ & $-0,6 \pm 1,3$ & $<0,001$ \\
L1-L4 T-score (SD) & $0,882 \pm 0,139$ & $0,984 \pm 0,154$ & $<0,001$ \\
Femoral neck BMD (g/cm $\left.{ }^{2}\right)$ & $-0,91 \pm 1,2$ & $0,0 \pm 1,3$ & $<0,001$ \\
Femoral neck T-score (SD) & $30(50 \%)$ & $14(23.3 \%)$ & $<0.001$ \\
Osteopenia or osteoporosis at femoral neck & $43(71.7 \%)$ & $19(31.7 \%)$ & $<0.001$ \\
Osteopenia or osteoporosis at L1-L4 & & & \\
\hline
\end{tabular}

- The odds-ratio of osteopenia-osteoporosis was 6.3.

- Low bone mineral mass concerned more frequently lumbar spine $(p<0.05)$ (table 2$)$.

Table 2: Frequency of osteopenia and osteoporosis at lumbar spine and at femoral neck ( $*$ : p between osteopenia and osteoporosis, and normal BMD)

\begin{tabular}{lccc}
\hline & L1-L4 & Femoral neck & $P^{*}$ \\
\hline Osteoporosis & $22(36.7 \%)$ & $5(8.3 \%)$ & 0.011 \\
Osteopenia & $21(35 \%)$ & $25(41.7 \%)$ & \\
Normal & $17(28.3 \%)$ & $30(50 \%)$ & \\
\hline
\end{tabular}

- Bone fracture was reported by 3 patients;

-one case of elbow fracture that occurred at 40 years of age, after 18 years of Sheehan syndrome duration, and the patient had an osteopenia

- two cases of metatarsal fractures that happened at 45 and 47 years of age, after respectively 14 and 15 years of Sheehan syndrome duration, and the patients had an osteopenia in one case and a normal BMD in the other case.

- Only six of the osteoporotic patients were treated with bisphosphonates. The others received only calcium and vitamin $D$ supplementation.

- Osteodensitometry was controlled in 15 cases after a mean duration of six years and showed an improvement of BMD in four cases.

\section{Conclusion}

Low bone mineral mass was very frequent in patients with Sheehan's syndrome. It concerned more frequently lumbar spine.

Treatment was insufficient. This is explained by the lack of social security coverage and the bad socio-economic conditions of these patients. 